\title{
Achados patológicos em caninos com displasia renal no Sul do Brasil ${ }^{1}$
}

\author{
Lorena L.B. Guimarães ${ }^{2}$, Matheus de O. Reis ${ }^{2}$, Kivia L. Hesse ${ }^{2}$, Fabiana M. \\ Boabaid $^{2}$, Saulo P. Pavarini ${ }^{2}$, Luciana Sonne ${ }^{2}$ e David Driemeier ${ }^{2 *}$
}

\begin{abstract}
Guimarães L.L.B., Reis M.O., Hesse K.L., Boabaid F.M., Pavarini S.P., Sonne L. \& Driemeier D. 2014. [Pathological findings in dogs with renal dysplasia in Southern Brazil.] Achados patológicos em caninos com displasia renal no Sul do Brasil. Pesquisa Veterinária Brasileira 34(12):1227-1230. Setor de Patologia Veterinária, Faculdade de Veterinária, Universidade Federal do Rio Grande do Sul, Av. Bento Gonçalves 9090, Bairro Agronomia, Porto Alegre, RS 91540-000, Brazil. E-mail: davetpat@ufrgs.br

Renal dysplasia results from a disturbance which occurs during nephrogenesis. The disease may be unilateral or bilateral, and leads to abnormal kidney differentiation leading to renal failure in young dogs. Several agents, including viruses, can cause the dysplasia till three months after birth, when the early nephron formation of the developing kidney is completed. Eleven renal dysplasia cases were diagnosed in 186 dogs with renal failure of 5,846 dogs necropsied in the Veterinary Pathology Sector, Federal University of Rio Grande do Sul, in the period 2002-2013. Tissue samples collected during necropsy were fixed in 10\% formaldehyde and stained with hematoxylin-eosin (HE) and Masson's trichrome protocol. Upon necropsy, kidneys were pale, smaller than normal, irregular and firm, and presented lower cortex diameter. Some exhibited cysts and whitish, parallel radially arranged striae in the medulla. Primary and secondary renal lesions were observed during histological examination. Primary lesions included fetal glomeruli and tubules, quantitative reduction in glomeruli, adenomatous tubules, and persistence of metanephric ducts. As for secondary lesions, Masson trichrome staining revealed intense interstitial fibrosis in all cases; furthermore, dilation of tubules and of Bowman's capsules, glomerular atrophy, and glomerulosclerosis were observed. Even though most cases were associated with breed, the results obtained indicate high prevalence of renal dysplasia in mixed-breed dogs, possibly because they were the majority of animals referred for necropsy. Although primary lesions were easily identified using HE, Masson's trichrome protocol is useful to characterize the extent of fibrosis.
\end{abstract}

INDEX TERMS: Dysplasia, kidney, dogs.

RESUMO.- A displasia renal resulta de um distúrbio na nefrogênese, com diferenciação anormal dos rins, podendo ser unilateral ou bilateral, e causa insuficiência renal em cães jovens. Qualquer agente agressor, incluindo vírus, pode causar a lesão até três meses após o nascimento, quando encerra o desenvolvimento embrionário do néfron. Foram diagnosticados 11 casos de displasia renal dentre 186 casos de cães com insuficiência renal de um total de 5.846 cães

\footnotetext{
${ }^{1}$ Recebido em 5 de novembro de 2014.

Aceito para publicação em 14 de novembro de 2014

${ }^{2}$ Setor de Patologia Veterinária (SPV), Faculdade de Veterinária (FAVET), Universidade Federal do Rio Grande do Sul, Av. Bento Gonçalves 9090, Bairro Agronomia, Porto Alegre, RS 91540-000, Brasil. *Autor para correspondência: davetpat@ufrgs.br
}

submetidos à necropsia no Setor de Patologia Veterinária da Universidade Federal do Rio Grande do Sul no período 2002-2013. Amostras teciduais coletadas na necropsia foram fixadas em $10 \%$ de formol e coradas com hematoxilina e eosina (HE) e tricrômico de Masson. Na necropsia, os rins estavam pálidos, diminuídos de tamanho, irregulares e firmes, com diâmetro da cortical diminuído. Alguns ainda eram císticos e apresentavam estriações brancacentas paralelas dispostas radialmente na medular. Ao exame histológico os rins exibiam lesões primárias e secundárias. As lesões primárias identificadas foram glomérulos e túbulos fetais, diminuição quantitativa de glomérulos, túbulos adenomatosos e persistência de ductos metanéfricos. Dentre as lesões secundárias, a coloração de tricrômico de Masson evi- 
denciou intensa fibrose intersticial em todos os casos. Além disso, foram observadas dilatação de túbulos e da cápsula de Bowman, atrofia glomerular e glomeruloesclerose. Ainda que a maioria dos casos tenha sido relacionada com linhagens raciais, esse trabalho demonstra uma alta frequência de diagnóstico de displasia renal em cães sem raça definida, possivelmente devido ao fato de que estes representam a maioria dos cães encaminhados ao laboratório. Apesar das lesões primárias serem facilmente identificadas pela coloração de hematoxilina eosina, a coloração de tricrômico de Masson auxilia na identificação da extensão da fibrose.

TERMOS DE INDEXAÇÃO: Displasia, rim, canino jovem.

\section{INTRODUÇÃO}

Define-se displasia renal como uma desorganização do parênquima devida a anormalidade na diferenciação (Maxie \& Newman 2007) em decorrência da nefrogênese anormal (Serakides 2010).

Classicamente, a doença pode ter causa hereditária ou adquirida. Cães de raças específicas como Lhasa Apso e Shih Tzu apresentam maior frequência de casos ( $O^{\prime}$ Brian et al. 1982) e foi observado que a variação alélica da cicloxigenase-2 está associada a casos de displasia renal em cães (Whiteley 2014). Além disso, infecções, especialmente virais, no trato urinário neonatal ou deficiência nutricional também podem resultar na doença (Maxie \& Newman 2007).

Em cães, a doença enquadra-se como um tipo de nefropatia juvenil (Lees 2007) que culmina com insuficiência renal entre os três meses e três anos de idade (Lees 2011). $\mathrm{Na}$ displasia renal os rins assemelham-se aos de cães idosos com nefropatia crônica e o diagnóstico é baseado em critérios histológicos que distinguem as lesões primárias das secundárias, incluindo respostas compensatórias à lesão (Picut \& Lewis 1987b). A displasia renal é uma doença complexa com alta variabilidade fenotípica (Whiteley 2011) que inclui como lesões primárias as estruturas inapropriadas ao estágio de desenvolvimento do animal ou o desenvolvimento de estruturas anormais (Maxie \& Newman 2007). 0 objetivo desse trabalho é descrever os aspectos anatomopatológicos e histoquímicos e a prevalência de cães diagnosticados com displasia renal pelo Setor de Patologia Veterinária da UFRGS.

\section{MATERIAL E MÉTODOS}

Realizou-se um estudo retrospectivo dos casos de necropsia de cães diagnosticados no Setor de Patologia Veterinária da Universidade Federal do Rio Grande do Sul (SPV-UFRGS) no período de 2002-2013. Dados como raça, sexo, idade e histórico clínico foram obtidos através das fichas clínicas dos animais.

Amostras teciduais, coletadas durante a necropsia, foram fixadas em solução de formalina $10 \%$, desidratadas em concentrações crescentes de álcool etílico, diafanizadas em xilol, incluídas em parafina, seccionadas em 3- $\mu$ m e coradas com pela técnica de hematoxilina e eosina (HE) e tricrômico de Masson.

\section{RESULTADOS}

De 5.846 cães submetidos à necropsia no Setor de Patologia Veterinária da Universidade Federal do Rio Grande do Sul (SPV-UFRGS) no período de 2002 - 2013 foram diag- nosticados 11 casos de displasia renal em 186 cães com insuficiência renal. Os cães não tinham parentesco e as informações acerca de raça, idade e sexo estão discriminadas no Quadro 1.

Na necropsia, os rins de todos os 11 cães estavam pálidos, diminuídos de tamanho, irregulares e firmes (Fig.1). Nos Casos 3 e 10 havia cistos de 0,1 a $0,5 \mathrm{~cm}$ de diâmetro. Nos Casos 1, 3, 4, 5, 6, 9 e 10 foi observada diminuição da espessura da cortical e em dois caninos havia estriações brancacentas paralelas dispostas radialmente na medular (Casos 1 e 11) (Fig.2). Dentre os 11 casos, seis cães apresentaram lesões de uremia; quatro cães tinham ulcerações gástricas (Casos 1, 5, 6 e 9); em dois cães havia ulcerações ventrais na língua (Casos 5 e 6); nos Casos 5 e 6 havia fragilidade óssea e, no Caso 5, ainda havia aumento de volume acentuado das paratireoides e deposição de material esbranquiçado, opaco e firme na superfície do endocárdio e sob a pleura parietal.

Ao exame histológico, os rins exibiam lesões primárias e secundárias. Em todos os casos a cortical apresentou diminuição de espessura. As lesões primárias identificadas foram glomérulos e túbulos fetais (Fig.3), caracterizadas por glomérulos diminutos e túbulos lineares com epitélio hi-

Quadro 1. Casos de insuficiência renal por displasia renal em cães necropsiados no Setor de Patologia Veterinária da UFRGS no período de 2002-2013

\begin{tabular}{lccl}
\hline Caso $\mathrm{n}^{\underline{0}}$ & Raça & $\begin{array}{c}\text { Idade } \\
\text { (meses) }\end{array}$ & Sexo \\
\hline 1 & Yorkshire & 2 & Macho \\
2 & Pitbull & 1,2 & Macho \\
3 & Poodle & 2 & Macho \\
4 & Pequinês & 9 & Macho \\
5 & Rhodesian & 36 & Macho \\
& Ridgeback & & \\
6 & SRD & 48 & Macho \\
7 & SRD & 12 & Macho \\
8 & SRD & 10 & Macho \\
9 & SRD & 0,5 & Macho \\
10 & SRD & 8 & Fêmea \\
11 & SRD & 4 & Fêmea
\end{tabular}

a Sem raça definida.

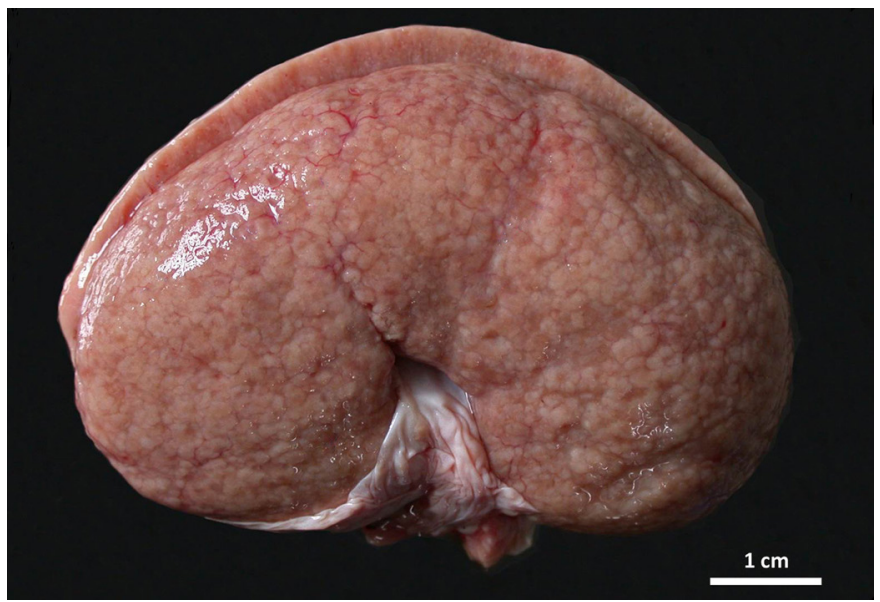

Fig.1. Displasia renal em caninos. Rim pálido, diminuído de tamanho e com a superfície subcapsular difusamente irregular no Caso 5. 


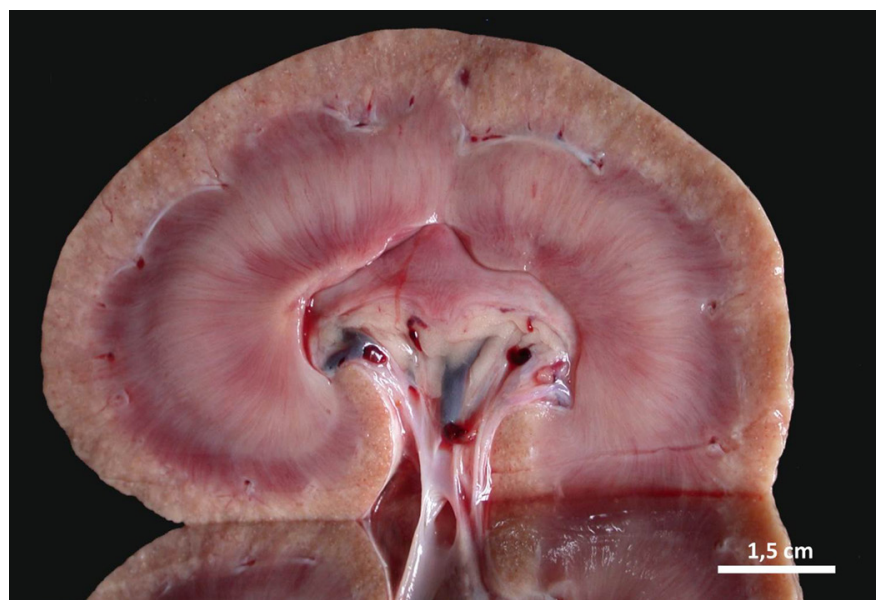

Fig.2. Rim do Caso 5. Cortical acentuadamente reduzida de tamanho e com inúmeros focos brancacentos e, na medular há estriações brancacentas paralelas dispostas radialmente.

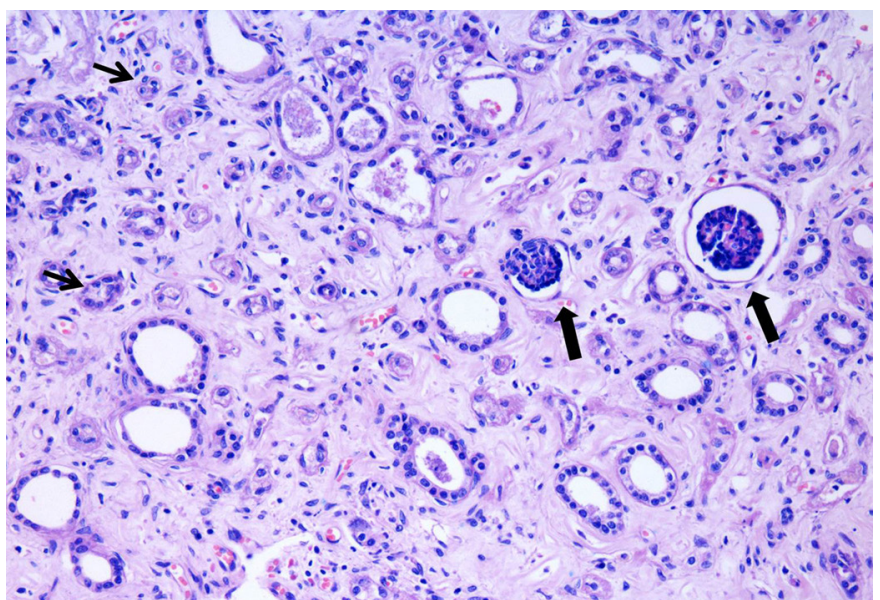

Fig.3. Displasia renal em caninos. Rim com glomérulos fetais (seta larga) e túbulos fetais (seta fina). HE, obj.20x.

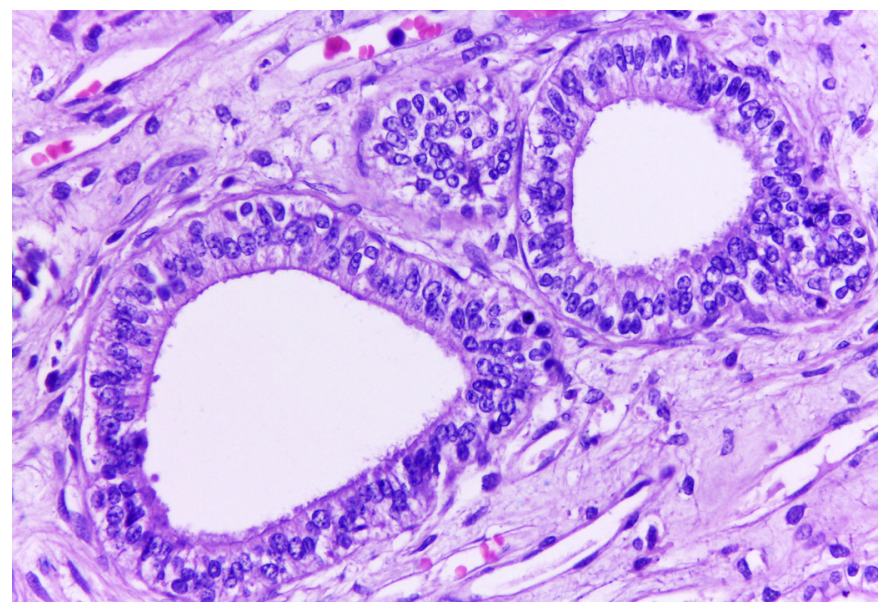

Fig.4. Displasia renal em caninos. Rim com ductos metanéfricos caracterizados por pseudoestratificação do epitélio colunar. HE, obj.40x.

percromático. Observou-se ainda diminuição quantitativa de glomérulos, persistência dos ductos metanéfricos com pseudoestratificação do epitélio colunar (Fig.4) e túbulos

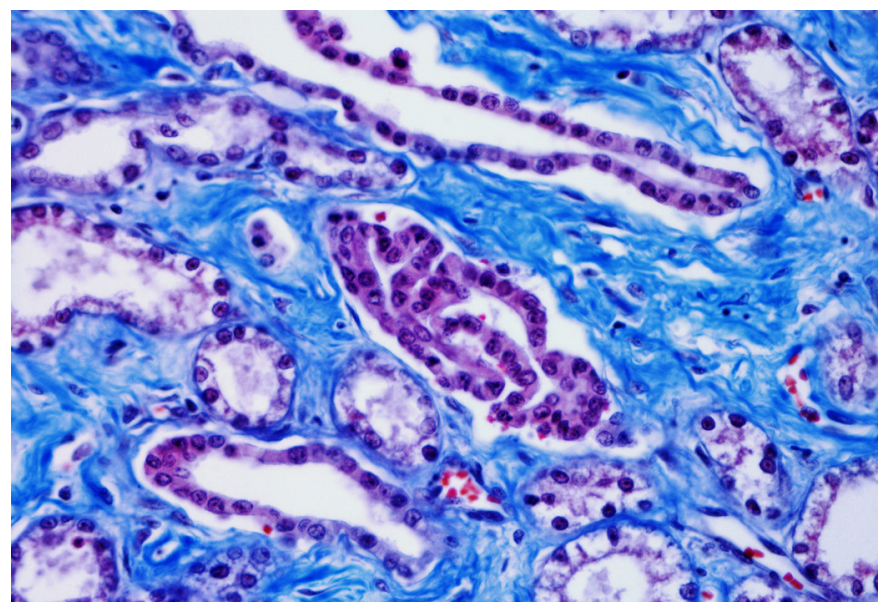

Fig.5. Displasia renal em caninos. Rim com túbulos adenomatosos entremeados por intensa proliferação de tecido conjuntivo fibroso (em azul). Tricrômico de Masson, obj.40x.

tortuosos de conformação semelhante a fita, denominados túbulos adenomatosos (Fig.5).

Tecido mesenquimal primitivo com aspecto mixomatoso foi observado apenas nos rins no Caso 9. Dentre as lesões secundárias havia fibrose intersticial em todos os casos, evidenciada pela coloração de tricrômico de Masson, dilatação de túbulos e da cápsula de Bowman, atrofia glomerular (Casos 3, 4, 5, 6, 10 e 11) e glomeruloesclerose (Casos 5, 6, 10 e 11) . Nos rins destes cães notou-se também infiltrado intersticial multifocal de linfócitos e plasmócitos, variando de discreto a acentuado. Em dois casos havia mineralização acentuada da membrana basal dos túbulos e da cápsula de Bowman (Casos 5 e 11) e quatro cães apresentaram degeneração tubular multifocal (Casos $4,5,10$ e 11 ).

Microscopicamente, os pulmões de oito caninos apresentaram alterações de pneumopatia urêmica, caracterizada por deposição de material basofílico granular (mineralização) nos septos interalveolares (Casos 1, 2 e 11), edema (Casos 4, 9 e 10), exsudação de fibrina (Casos 2, 5 e 11) e infiltrado inflamatório constituído por neutrófilos (apenas no Caso 11) e linfócitos (Casos 4 e 8) nos alvéolos. No Caso 5 foi observada hiperplasia e vacuolização do epitélio das paratireoides, além de mineralização de cardiomiócitos, dos septos alveolares e da musculatura esquelética intercostal. E, nos Casos 1, 2 e 11 havia hiperparatireoidismo secundário com osteodistrofia fibrosa associado à displasia renal.

\section{DISCUSSÃO}

Fatores genéticos e ambientais são determinantes nos casos de displasia renal. Testes genéticos específicos, que estão em fase de desenvolvimento, podem contribuir para seu diagnóstico e controle em raças e famílias com alto risco da doença (Whiteley et al. 2011). Contudo este trabalho demonstra a variedade de raças e cães sem raça que podem ser acometidos pela displasia renal, dificultando a prevenção de novos casos.

A maioria dos cães desenvolve sinais clínicos até os 12 meses de idade, com progressão rápida para a morte 
(O'Brien et al. 1982). Contudo Schulze et al. (1998) descreveram um caso de displasia renal em um cão de 3 anos. Neste trabalho foi confirmada a doença em um canino de três anos de idade e também em um cão de quatro anos.

No exame macroscópico as lesões de displasia renal são indistinguíveis das nefropatias crônicas de cães (Picut \& Lewis 1987b). São descritos rins pequenos, firmes e pálidos, frequentemente lobulados. Embora os casos relatados não apresentavam lobulação, os caracteres morfológicos condizem com displasia renal. Há casos de severa diminuição do diâmetro da cortical (O'Brian et al., 1982) e a relação corticomedular pode estar reduzida, uniformemente ou de forma segmentar. Nos cães deste relato a cortical apresentava espessura difusamente diminuída e, concordando com Picut \& Lewis (1987b) muitas vezes com feixes fibrosos brancacentos estendendo-se da cortical à medular. Podem estar presentes múltiplos cistos, tanto na cortical, quanto na medular (O'Brian et al. 1982, Picut \& Lewis 1987b), como os observados nos casos descritos neste trabalho.

Em todos os cães deste trabalho foram observados glomérulos e túbulos imaturos ou fetais ao exame histológico da cortical, alterações definidas como lesão primária (Picut \& Lewis 1987b), além de diminuição quantitativa de glomérulos.

Na displasia renal são descritas alterações secundárias, degenerativas e inflamatórias. As alterações secundárias incluem hiperplasia e hipertrofia de glomérulos e túbulos. Lesões compensatórias não foram observadas nos cães do estudo, mas houve dilatação dos túbulos e da cápsula de Bowman, atrofia glomerular e glomeruloesclerose. Lesões degenerativas e inflamatórias, incluindo fibrose e nefrite intersticial, são descritas na doença (Picut \& Lewis 1987b). Embora tenha sido observada glomeruloesclerose em apenas quatro cães, havia infiltrado inflamatório e fibrose em todos os casos. Segundo Picut \& Lewis (1987b), estroma mesenquimal indiferenciado com metaplasia óssea e cartilaginosa está frequentemente presente, no entanto não foi identificado nos caninos deste trabalho.

Houve lesões extra-renais de uremia em seis casos descritos, que incluíram ulcerações na mucosa gástrica e na língua, além de mineralização em septos interalveolares, endocárdio e músculo intercostal. O’Brian et al. (1982) descreveram lesões multissistêmicas consistentes com insuficiência renal crônica, incluindo gastropatia e mineralização urêmica, estas também observadas por Abraham et al. (2003) e Volkweis et al. (2012).

Doenças secundárias à displasia renal podem ser encontradas, como hiperparatireoidismo com osteodistrofia fibrosa (Brum et al. 2008) e neste trabalho foi diagnosti- cado hiperparatireoidismo secundário renal em três casos, com osteodistrofia fibrosa.

Ainda que os casos descritos na literatura tenham sido relacionados com linhagens raciais (O'Brian et al. 1982) esse trabalho demonstra significativa frequência de diagnóstico de displasia renal em cães sem raça definida, possivelmente devido ao fato de que estes representam a maioria dos cães encaminhados ao Setor de Patologia. A faixa etária observada, composta por animais muito jovens em sua maioria, corrobora para doença de origem hereditária, como descrito no trabalho de Picut \& Lewis (1987a). Além disso, apesar das lesões primárias serem facilmente identificadas pela coloração de hematoxilina e eosina, a coloração de tricrômico de Masson auxiliou na identificação da extensão da fibrose.

\section{REFERÊNCIAS}

Abraham L.A., Beck C. \& Slocombe R.F. 2003. Renal dysplasia and urinary tract infection in a Bull Mastiff puppy. Aust. Vet. J. 81:336-339.

Brum K.B., Juliano R.S., Souza M.A., Fleury L.F.F. \& Damasceno A.D. 2008. Renal dysplasia in a Boxer dog: Case report. Arq. Bras. Med. Vet. Zootec. 60:1084-1088.

Lees G.E. 2007. Juvenile and familial nephropathies, p.79-86. In: Elliott J. \& Grauer G.F. (Eds), BSAVA Manual of Canine and Feline Nephrology and Urology. $2^{\text {nd }}$ ed. Gloucester, England.

Lees G.E. 2011. Congenital kidney diseases, p.568-576. In: Bartges J. \& Polzin D.J. (Eds), Nephrology and Urology of Small Animals. John Wiley \& Sons, New Jersey.

Maxie M.G. \& Newman S.J. 2007. The urinary system, p.425-442. In: Maxie M.G. (Ed.), Jubb, Kennedy and Palmer's Pathology of Domestic Animals. Vol.2. $5^{\text {th }}$ ed. Saunders Elsevier, Philadelphia.

O’Brien T.D., Osborne C.A., Yano B.L. \& Barnes D.M. 1982. Clinicopathologic manifestations of progressive renal disease in Lhasa Apso and Shih Tzu dogs. J. Am. Vet. Med. Assoc. 180:658-664.

Picut C.A. \& Lewis R.M. 1987a. Comparative pathology of canine hereditary nephropathies: An interpretative review. Vet. Res. Commun. 11:561-581.

Picut C.A. \& Lewis R.M. 1987b. Microscopic features of canine renal dysplasia. Vet. Pathol. 24:156-163.

Schulze C., Meyer H.P., Blok A.L., Schipper K. \& Van den Ingh T.S.G.A.M. 1998. Renal dysplasia in three young adult Dutch Kooiker dogs. Vet. Q. 20:146-8.

Serakides R. 2010. Sistema urinário, p.291-336. In: Santos R.L. \& Alessi A.C. (Eds), Patologia Veterinária. Roca, São Paulo.

Volkweis F.S., Almeida A.M.S., Wong L., Mulinari F. \& Santos Júnior H.L. 2012. Displasia renal em cão da raça Rotweiller. Arq. Bras. Med. Vet. Zootec. 64:1511-1514.

Whiteley M.H. 2014. Allelic variation in the canine Cox-2 promoter causes hypermethylation of the canine Cox-2 promoter in clinical cases of renal dysplasia. Clin. Epigenetics 6:7.

Whiteley M.H., Bell J.S. \& Rothman D.A. 2011. Novel allelic variants in the canine cyclooxygenase-2 (Cox-2) promoter are associated with renal dysplasia in dogs. PlosOne 7:e16684. 